\title{
Tiempo y escuela. Aportación desde la cronobiología y cronopsicología a la Sociología de la Educación. Anotaciones para la reflexión
}

\author{
Time and School. Contribution from Chronobiology and Chronopsychology \\ to the Sociology of Education. Notes for Reflection
}

\section{Santiago Estaún Ferrer ${ }^{1}$}

\begin{abstract}
Resumen
La preocupación por una correcta organización de los horarios escolares se centra en el qué (curriculum), el cómo (metodología) y en el cuándo hay que enseñar, pero este cuando se centra en la mayoría de las veces en cómo distribuir las materias a lo largo de la jornada, o en el tipo de jornada escolar. La cronobiología y cronopsicología centran su mirada en la organización de la propia actividad del ser vivo, la persona, y aportan conocimientos sobre los momentos óptimos de determinadas actividades y la conveniencia de respetarlas dentro de unos márgenes adaptativos, como son la activación y el reposo / sueño, el ritmo atencional, la memoria, y la actividad intelectual en su conjunto, mostrando la complejidad de su integración respetando el reloj biológico a la par que integrándolo socioculturalmente, de una persona en continuo cambio.
\end{abstract}

Palabras clave

Cronobiología, cronopsicología, ritmos atencionales, ritmos memoria, sueño, hemisferio derecho, hemisferio izquierdo.

\section{Abstract}

The concern for a correct organization of school schedules focuses on the what (curriculum), the how (methodology) and when to teach, but this when it focuses most of the time on how to distribute the subjects to the throughout the day, or in the type of school day. Chronobiology and chronopsychology focus their gaze on the organization of the activity of the living being, the person, and provide knowledge about the optimal moments of certain activities and the convenience of respecting them within adaptive margins, such as activation and rest /sleep, attentional rhythm, memory, and intellectual activity as a whole, showing the complexity of their integration respecting the biological clock as well as integrating it socio-culturally, of a person in continuous change.

\section{Keywords}

Chronobiology, chronopsychology, attention rhythms, memory rhythms, sleep, right hemisphere, left hemisphere.

\section{Cómo citar/Citation}

Estaún Ferrer, Santiago (2021). Tiempo y escuela. Aportación desde la cronobiología y cronopsicología a la Sociología de la Educación. Anotaciones para la reflexión. Revista de Sociología de la Educación-RASE, 14 (3), 244-255. http://dx.doi.org/10.7203/RASE.14.3.21631. 


\section{Introducción}

Tiempo y escuela tienen una larga interrelación. Unas veces bajo el prisma de la fatiga escolar; otras, de cómo secuenciar el currículum docente; otras, sobre cuántas horas lectivas anuales y/o cómo distribuirlas a lo largo del año académico; otras, si es preferible jornada intensiva o partida; etc. Sin lugar a dudas esta última interrelación es la que hoy está siendo debatida, con razones que apelan a la fatiga del alumno; a un mejor rendimiento académico; a posibilitar tiempo para la actividad extraescolar al alumno; a facilitar la conciliación familiar; etc. Todas estas aproximaciones y perspectivas sobre la relación del tiempo y la escuela indican la complejidad de hablar sobre los horarios escolares sin tener presentes aspectos relevantes de la dimensión social: el mundo laboral (incluyendo al profesorado), la actividad social y familiar, cultural y de ocio. Abre, además, la posibilidad de olvidar quién es el centro de la actividad educacional: una persona en pleno desarrollo.

El interés de la relación tiempo y escuela se remonta como mínimo a 1885 con el trabajo que presentó A. Key (citado por Crépon, 1983) al comité escolar de la Asociación de Médicos Suecos, proponiendo una distribución del tiempo según las actividades a realizar por el alumno desde los 6 a los 17 años, incluyendo el tiempo que deberían dedicar al sueño (11 horas a los 6 años y 8:30 a los 17).

El tema lo retoma el Prof. Bojlen en 1954, al presentarlo en la Conferencia Europea de Higiene y de Medicina Escolar, con algunas modificaciones en la distribución del tiempo atribuido a las diferentes actividades del niño, pero incluyendo también las horas necesarias dedicadas al sueño, según la edad del alumno (citado por Crépon,1983)

En 1976 el Dr. Guy Vermeil publica La fatigue à l'école en donde revisando diversos estudios sobre fatiga escolar y entre otros, el informe realizado por el profesor Robert Debré y el Dr. Daniel Douady en 1962 para el Conseil de la Recherche Pédadogique de Francia, reclama y pide no sólo que el niño sea el centro de la organización temporal de la escuela sino también que se tenga en cuenta las diferencias individuales.

El interés de estas propuestas realizadas desde la medicina era no sólo por la atención prestada al sueño, sino también, y quizás principalmente, a la distribución de horas diarias, semanales e incluso anuales que se recomendaba dedicar a las diferentes tareas de estudio, actividades libres, tiempo dedicado a la alimentación, al trabajo escolar en clase y en casa, etc., según la edad de los escolares. Todo ello y también la sugerencia de pausas en determinados momentos de la actividad escolar sin mayor concreción, sí que fueron y han sido útiles para cómo organizar la actividad escolar de conformidad con las teorías del aprendizaje y el desarrollo de estrategias del aprender.

Sin embargo, los biólogos o mejor, la Cronobiología y la Cronofarmacología inicialmente y, con posteridad, la Cronopsicología han complementado dicha aproximación a la distribución temporal del alumno, al evidenciar la perspectiva cíclica de toda la actividad del ser vivo y por supuesto del hombre. Así en la actualidad se sabe que la eficacia de un medicamento depende no sólo de su composición química, sino también de la hora en que se administra y que la toxicidad de una sustancia depende también del momento de ingerirla o de la ocurrencia de la herida. Así se conoce la influencia de la temperatura corporal, la actividad hormonal (cortisol y melatonina, por ejemplo), actividad y reposo, la menstruación, entre otras funciones del organismo. 
De forma semejante puede decirse de la actividad cíclica o rítmica de nuestro cerebro y en general de la actividad física e intelectual del hombre: existen momentos máximos y mínimos en nuestra actividad que se repiten de forma periódica o cíclica los cuales pueden ser indicadores, de forma genérica, del momento oportuno que nos permita obtener el mejor rendimiento en nuestra actividad, y no sólo de nuestro potencial biopsicológico, sino también de nuestra salud y sensación de bienestar.

Pero los cambios que de forma rítmica se observan en todo el universo físico, biológico, psicológico y sociocultural, presentan una escala de intervalos diferenciados por su duración. Así se puede hablar de ritmos o ciclos infradianos, circadianos y ultradianos, según sea su duración o si se prefiere la frecuencia de repetición de menor a mayor respectivamente.

Los biorritmos poseen las características: a) de ser internos y estar sometidos a un cierto control genético, principalmente los ritmos ultradianos; b)de requerir la presencia de determinados estímulos externos para su activación durante el desarrollo de la persona; y c) los parámetros que los definen, como el ciclo, o la fase, etc., pueden ser modificados o condicionados por determinados factores ambientales.

El origen de la actividad rítmica tanto biológica como psicológica puede ser atribuida tanto a factores endógenos y hereditarios como a factores externos y condicionantes. En general, los ritmos biopsíquicos ultradianos son de origen endógeno (temperatura del cuerpo, ritmo cardíaco, etc.), mientras que los circadianos e infradianos son más susceptibles a factores exógenos y pueden ser condicionados por el ambiente.

La maduración y el desarrollo es uno de estos factores intervinientes en el funcionamiento de los biorritmos fisiológicos y psicológicos. Son aspectos a considerar puesto que, dentro de unas pautas generales de desarrollo y maduración, como han mostrado Piaget, Vygotsky, Montessori, Freinet y otros, no todos llegan a desarrollar sus capacidades físicas, psicológicas y cognitivas al mismo tiempo biológica y psicológicamente considerado. Es conocido que la maduración de las niñas es superior o más rápida que la de los niños en múltiples funciones.

Un ejemplo de la incidencia del factor maduración puede ser útil: la actividad circadiana de sueño/ vigilia. Desde el nacimiento el ser humano alterna periodos de sueño con los de vigilia. Esta alternancia es innata y se desarrolla y establece ciclos de sueño y vigilia cada vez más amplios según su desarrollo físiológico. Dos aspectos deben ser considerados:

El primero, el proceso de maduración de la persona, y el segundo las características de dicho ritmo inicialmente ultradiano para convertirse en circadiano a lo largo del proceso de desarrollo. Las observaciones realizadas, permiten indicar que el total de tiempo dedicado al sueño va de las 16/18 horas durante los primeros días hasta una cierta estabilización alrededor de las 8 horas a partir de la adolescencia y juventud. (Cfr. Gráfico 1). 


\section{Gráfico 1. Evolución según edad de la duración diaria de sueño y de la proporción de sueño lento (SOL) y sueño paradójico o REM (SOR)}

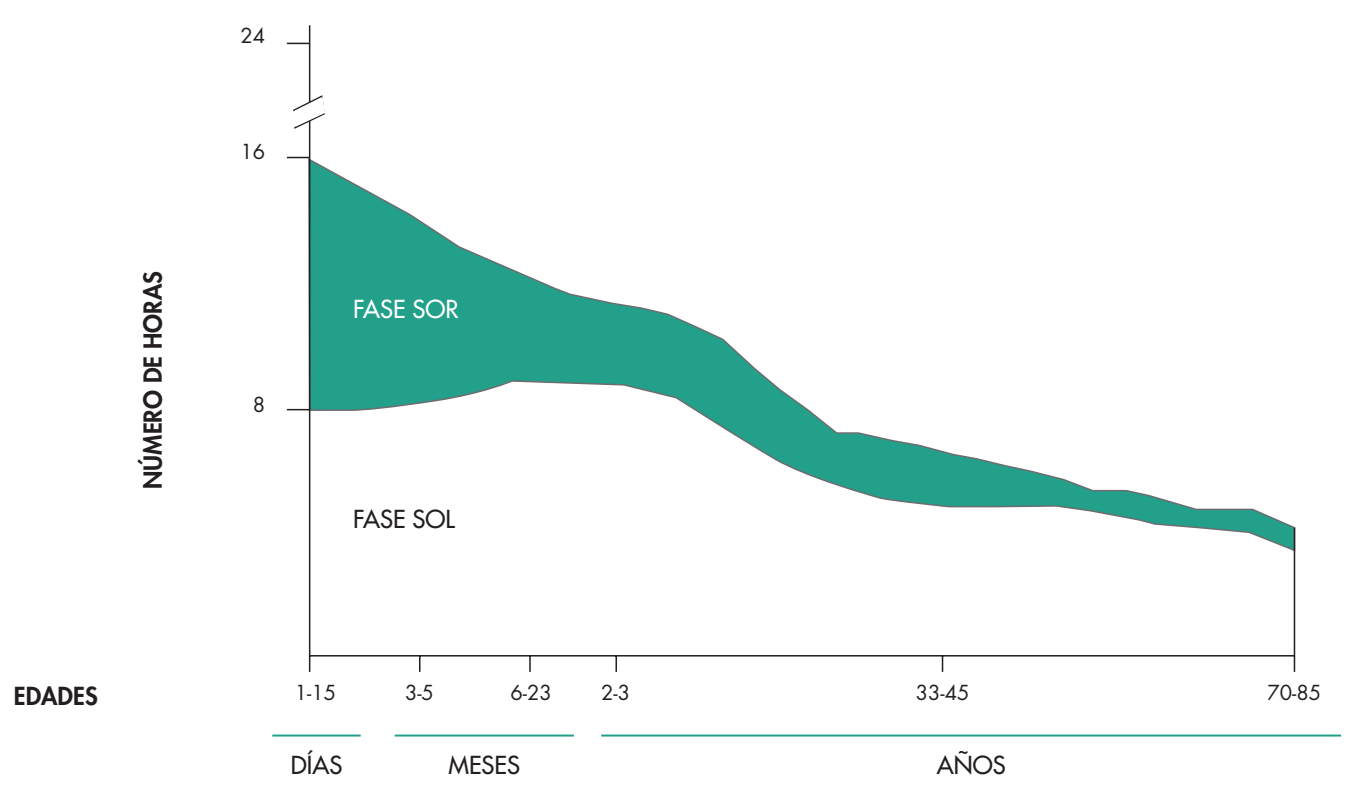

Fuente: adaptado de Roffwarg et al., (1966).

Sin embargo, la importancia de esta actividad cíclica radica en toda la actividad coordinada que realiza el organismo, con su denominado reloj biológico, en el desarrollo madurativo.

El segundo aspecto a considerar es el efecto del factor maduración y/o desarrollo en la disminución de la fase de sueño REM, sueño al que se le atribuye la consolidación de aprendizajes realizados durante el periodo o fase activa de la persona.

Los múltiples trabajos realizados muestran la característica de la existencia de una actividad fundamental del organismo que se realiza en ciclos o periodos más cortos (de 90 a 110 minutos), denominada ciclo básico de actividad y reposo (BRAC por sus iniciales inglesas) que se manifiesta durante el sueño con la alternancia de sueño lento y sueño REM (ambos constituyen un ciclo o periodo) y durante el día con la alternación de las correspondientes fases de activación y reposo en el desarrollo de las actividades físicas, psíquicas y cognitivas.

Un ejemplo de la interrelación de estas actividades es el jetlag, o desfase horario. Cuando se realiza un viaje transcontinental, la adaptación al nuevo escenario ambiental requiere de un cierto tiempo e incluso días tanto en la actividad física, como en la mental, según se realice el viaje de este a oeste (Paris-Nueva York) o en sentido contrario de oeste a este. Otros ejemplos se hallan en las experiencias realizadas por el equipo de Golombek (2007) citado por Acuña (2018), o las conocidas experiencias de aislamiento sensorial realizadas por Siffre en 1963 o Mills (1964) durante 58 y 105 días respectivamente en las que el ritmo de vigilia/sueño muestran un ciclo próximo a las 25 horas, que es algo superior a las 24 horas del ciclo natural establecido, es decir, el organismo mantiene su regulación cíclica endógena aunque se adapta dentro de un límite al factor exógeno luz/obscuridad de la naturaleza. Semejantemente se podría decir del cambio de hora en primavera y en otoño, aunque los efectos se notan en menor escala o incluso del desfase de ritmos que ocurre durante el fin de semana.

Por otra parte, Thatcher en 1987, mediante exploración electroencefalográfica en un estudio con 577 participantes desde los 2 meses hasta los 16 años, señala el desarrollo asimétrico de los dos hemisferios 
cerebrales (Cfr. Gráfico 2). Esta asimetría es coherente con la correlación de la actividad bioeléctrica del hemisferio derecho con actividades de tipo visual, espacial, holístico, concreto, intuitivo y artístico, y las de tipo verbal-lingüístico, analítico, abstracto, matemático y lógico con la del hemisferio izquierdo (Meccaci, 1984).

\section{Gráfico 2. Porcentaje del desarrollo de los hemisferios cerebrales según valores de fase EEG en el adulto. Las barras punteadas representan el hemisferio izquierdo, las blancas el hemisferio derecho}

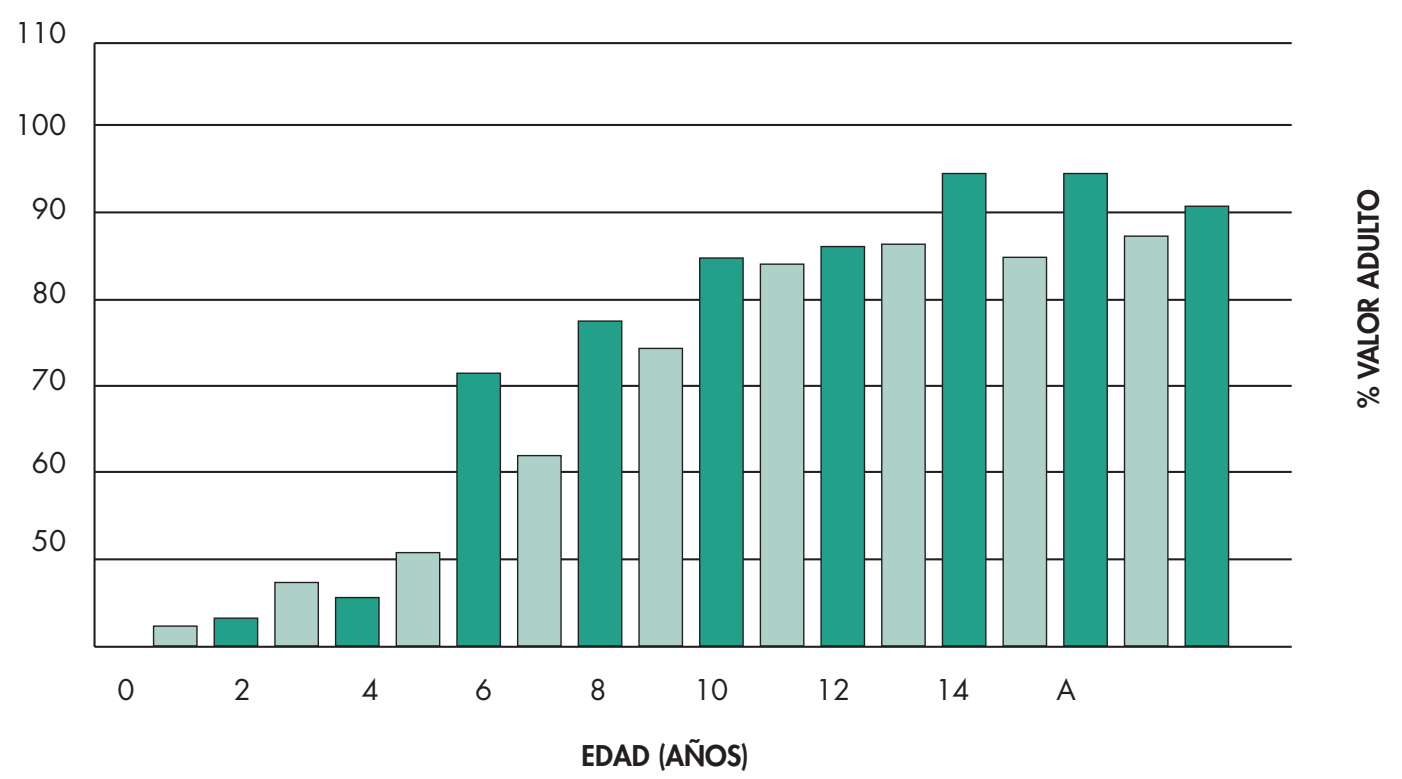

Fuente: adaptado de Thatcher et al., 1987.

Klein y Armitage habían publicado en Science unos años antes (1979) un experimento en el que los resultados indican no solamente la actividad diferenciada de ambos hemisferios, sino también dos periodos con fases antagónicas. El trabajo experimental realizado, consistía en dos tareas: una de tipo verbal y la otra, de tipo espacial. Se ejecutaban cada 15 minutos a lo largo de 8 horas, iniciándose a las 9:00 h. de la mañana. El análisis estadístico de Fourier, muestra un ciclo de 96 minutos y las fases óptimas de cada tarea se hallan opuestas. (Cfr. Gráfico 3). Estos resultados son indicativos de un ritmo circadiano cuyo origen se puede considerar endógeno. 


\section{Gráfico 3. Representación de resultados obtenidos por Klein y Armitage (1979), en una tarea verbal (trazo continuo) y otra espacial (trazo puntillado)}

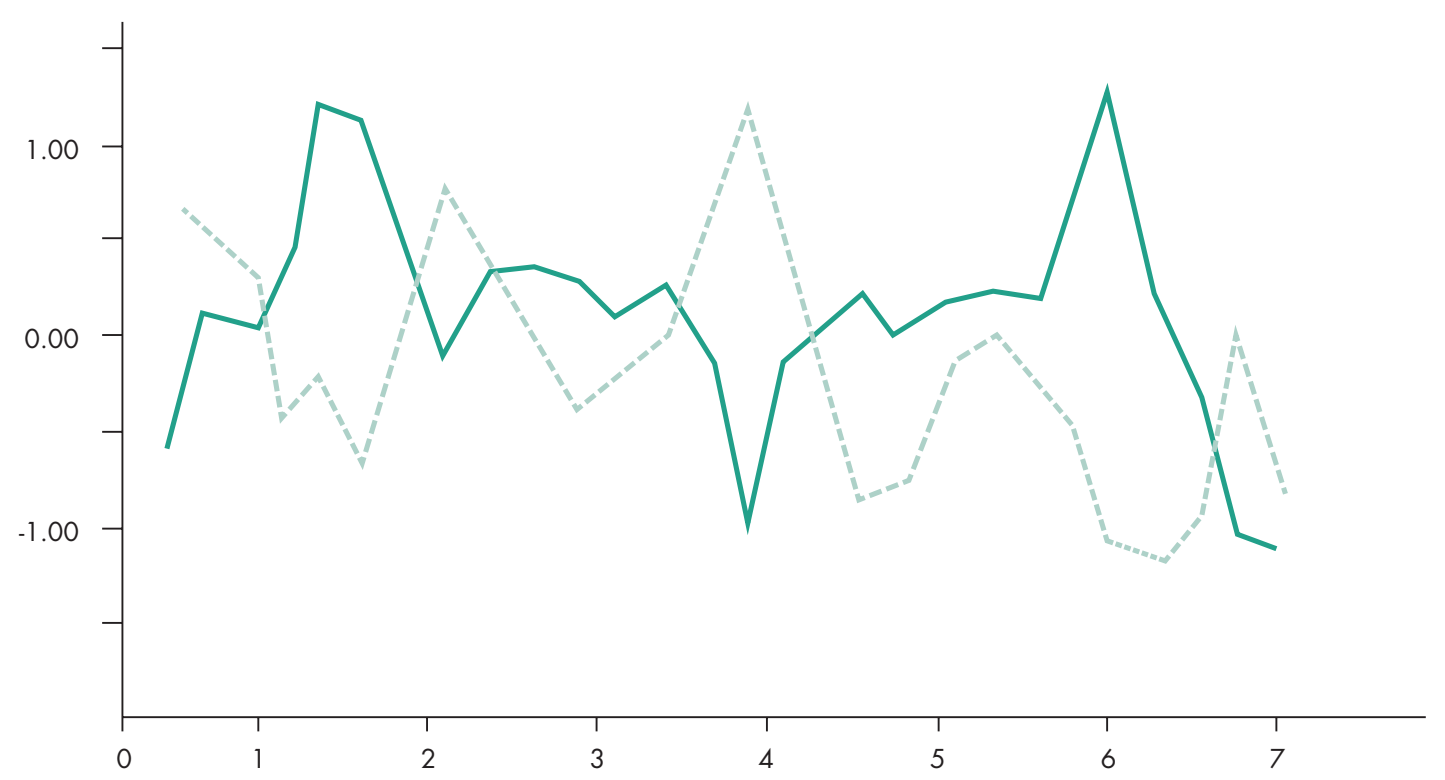

Sin embargo, experiencias aportadas por Beugnet-Lambert, Lancry y Leconte (1988) y el mismo Leconte, P. (1989) con experiencias de tareas de memoria semántica y gráfica realizadas cada 25 minutos, muestran que esta doble ritmicidad hemisférica puede estar modulada por características de personalidad como es la Dependencia de Campo, pero no por la característica Independencia de Campo. Aspectos que incrementan la complejidad de nuestro reloj interno biológico

Si el estudio del sueño y del desarrollo asimétrico cerebral aporta información útil a tener presente al organizar la actividad educativa, no es de menor importancia el conocimiento de los efectos de la ingesta alimenticia en edad escolar. Sin entrar en detallar experiencias hay que recordar a Binet, creador del test de inteligencia Binet-Simon, que a inicios del siglo xx ya indicó la necesidad de una alimentación adecuada para obtener un rendimiento óptimo de los escolares. Pero ha sido Hubert Montagner, de la Université de Besançon, con su equipo de colaboradores, quien desde 1979 ha trabajado de forma continuada el tema de una correcta distribución de la ingesta alimentaria en edad escolar, publicando resultados de experiencias realizadas con grupos de alumnos a quienes se les administraba diferente cantidad de calorías en el desayuno y se controlaba su capacidad de atención y rendimiento observando un déficit en aquellos cuyo desayuno había sido inferior a 50 calorías.

Si la cronobiología aporta información suficiente sobre los ciclos de actividad del organismo humano y los problemas que suscitan no respetarlos adecuadamente, habida cuenta de su capacidad de adaptación a estímulos externos (naturales o sociales) o internos (estados emocionales, presión estresante, características personales, etc.), la cronopsicología aporta por su parte aquellos conocimientos sobre los momentos óptimos de aquellos procesos que influyen en el rendimiento académico como son la atención, la memoria, la inteligencia.

La atención es un tema de particular interés no sólo para todo profesor sino también para una organización laboral optimizadora, puesto que no hay actividad humana sin un mínimo de atención sostenida. Tres son los aspectos que se pueden considerar en el tema de la atención: su ritmicidad, factores (externos o internos) que intervienen en ella y la edad. 
Las tareas que se han utilizado para el estudio de la atención han sido diversas y relativamente sencillas, aunque requerían de una atención continuada, unas veces en forma de tachado de letras o figuras y otras en forma de sincronización motriz; unas veces en ambiente escolar, otras en ambiente laboral (ritmo de trabajo en cadena).

En el conjunto de resultados de los trabajos realizados desde Gates (1916) por Pöppel et al., (1970), Hughes y Folkard (1976), Testu (1989), Beugnet-Lambert (1985), Canals et al., (1989), Leconte (2011) entre otros, tanto si se atiende a la edad escolar como adulta, la atención muestra una ritmicidad circadiana consistente en un incremento paulatino de la atención hasta media mañana una pausa (denominada postprandial) y un nuevo incremento por la tarde con un descenso vespertino. La duración de dicho ciclo oscila entre 90 y 120 minutos, para un adulto, y en función de la edad para un niño.

Esta ritmicidad circadiana de la atención está sujeta a influencias externas e internas al sujeto. La incidencia de los factores externos tanto naturales como sociales se hallan modulados por el conjunto de variables denominadas diferencias individuales. Blake, en 1971, ya indicaba que las personas introvertidas obtenían mejores resultados por la mañana, que las extrovertidas y su inversa por la tarde, y Horne y Östberg (1977) y Horne et al., (1980) indican que matutinos y nocturnos siguen una pauta semejante. Sin embargo, Akerstedt y Fröberg (1976) no hallan estos resultados en una tarea de detección de estímulos auditivos, lo cual puede ser interpretado que determinadas tareas no son moduladas por las características individuales.

Hederich et al., (2003) se propone estudiar la incidencia del factor entorno ecocultural (urbano y rural) y la jornada escolar a la que asiste (mañana-tarde) y de las variables individuales (estilo cognitivo y cronotipo). Un análisis detallado de los resultados indica que los mejores resultados atencionales se obtienen hacia la mitad de la mañana y de la tarde, pero si se considera el tipo de jornada (mañana o tarde) los resultados señalan diferencias a considerar como son: un mayor nivel de activación en la jornada de la tarde con incremento de errores, y una mayor estabilidad en la cantidad de respuestas correctas, respecto de la jornada matutina. Por el contrario, no parece que ni el cronotipo, ni el estilo cognitivo, incidan significativamente en el momento óptimo de activación en la mañana y en el de la tarde.

Testu y su equipo realiza en 1993 un estudio de los ritmos de la atención, de la capacidad de estructurar el espacio y de lógica de los escolares de 6-7 y de 10-11 años en cuatro centros escolares europeos (Francia, Alemania, Inglaterra y España) con jornada (continua o partida) y horarios escolares diferentes (diversidad de horas de inicio y finalización) y semanal. Los resultados obtenidos le permiten indicar que el ritmo circadiano de la atención mantiene el ciclo clásico de incremento por la mañana, pausa a mediodía y nuevo incremento por la tarde con independencia del tipo de jornada escolar (Gráfico 4), aunque siempre existe un intervalo de fluctuación del ciclo habida cuenta de la edad y del contexto sociocultural y geográfico, que no permite concretar el momento óptimo de mañana y el de la tarde. 


\section{Gráfico 4. Resultados atencionales obtenidos en Alemania, España, Francia e Inglaterra, en escolares de 6-7 años y de 10-11 años}
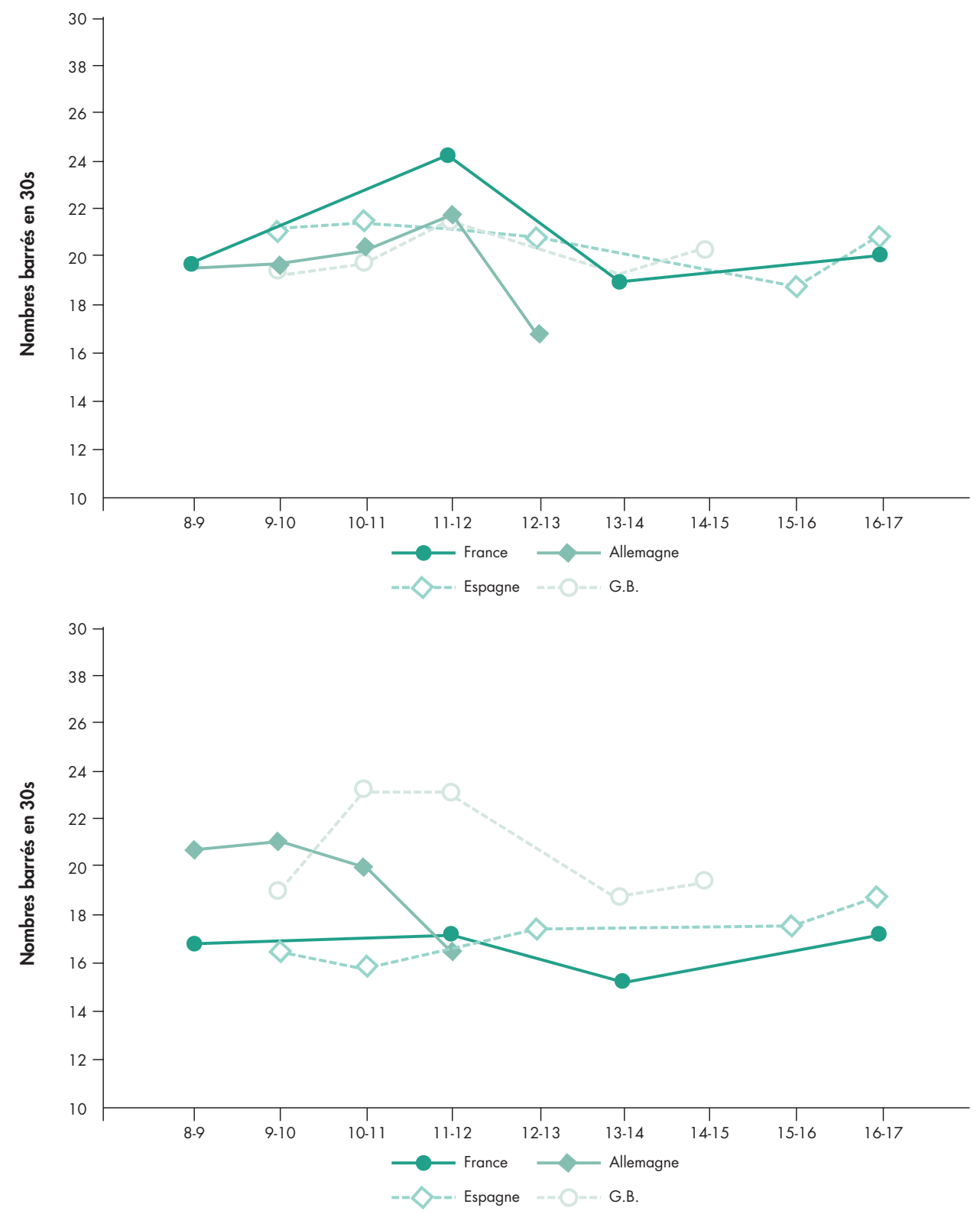

Fuente: tomado de Testu, 1993.

Una anécdota a propósito de la ritmicidad circadiana de la atención es el comentario de una profesora de matemáticas que tenía tres grupos seguidos por la mañana: «ahora entiendo por qué los alumnos del primer grupo tienen peores notas que los del tercer grupo cuando están más quietos y atentos los del primer grupo». Es fácil confundir la atención con la inacción.

El conjunto de datos comentados permite pensar que la atención manifiesta una ciclicidad circadiana, pero que dicha ritmicidad está influenciada no sólo por factores externos, naturales o sociales, sino también por los individuales y por el tipo de estímulo o tarea a realizar.

La memoria posee múltiples aspectos: semántica, episódica, primaria, secundaria, aspectos neurofisiológicos, evolutiva, inmediata y diferida. La cronopsicología se ha centrado en estas dos últimas acepciones. Gates (1916), citándolo nuevamente, ya indicaba que la memoria inmediata obtenía mejores resultados 
por la mañana que por la tarde. Folkard y sus colaboradores, (1977) publican los resultados obtenidos con escolares, de la tarea consistente en la lectura en voz alta y memorización de un texto. El control se realiza mediante cuestionario de elección múltiple de forma inmediata después de la lectura y diferido una semana después modificando el horario inicial a la mitad de cada grupo. La conclusión es que la memoria inmediata presenta mejores resultados por la mañana que por la tarde, pero no así la memoria diferida (Tabla I).

Tabla I. Respuestas correctas del recuerdo de un texto leído

\begin{tabular}{cccc}
\hline $\begin{array}{c}\text { HORA } \\
\text { LECTURA TEXTO }\end{array}$ & $\begin{array}{c}\text { RECUERDO } \\
\text { INMEDIATO }\end{array}$ & $\begin{array}{c}\text { RECUERDO DIFERIDO } \\
\text { MISMA HORA }\end{array}$ & $\begin{array}{c}\text { RECUERDO DIFERIDO } \\
\text { DIFERENTE HORA }\end{array}$ \\
\hline $9 \mathrm{~h}$. & 16,8 & 12,9 & $(15 \mathrm{~h}) 12,7$. \\
\hline $15 \mathrm{~h}$ & 15,2 & 14,0 & $(9 \mathrm{~h}) 14,0$. \\
\hline
\end{tabular}

Fuente: Basado en Folkard et al., (1977).

En 1980, Folkard et al., analizan estos resultados y observan que en la memoria inmediata muestra que la memoria inmediata muestra mayor número de elementos poco significativos por la mañana, mientras que por la tarde predominan los aspectos significativos de la narración. En la memoria diferida, por el contrario, existe una estabilidad de recuerdo de los datos importantes con independencia de la hora de aprendizaje.

Sáiz (1988) y Sáiz y Estaún (1990), publican los resultados obtenidos en un estudio de memorización de diversas tareas (memoria de dígitos, de narración y de imágenes) y en 5 momentos del día (8:00, 12:00, 16:00, 20:00 y 24:00 horas). Los indican un ritmo circadiano en ambas situaciones, inmediata y diferida, aunque sugieren que en la memoria inmediata de material visual y la de texto en memoria diferida incrementan el recuerdo al final de la jornada. Estos resultados sugieren una relación entre memoria y sueño.

En este sentido, son conocidos los trabajos realizados por Bloch y colaboradores $(1966,1970)$ y Bloch (1973) manipulando el nivel de activación del sistema nervioso, y los de Hennevin et Leconte (1977) mediante la privación selectiva del sueño paradójico. Y a pesar que los investigadores utilizan diferentes tareas y estrategias experimentales sí se observa que dicha privación del sueño REM no parece afectar al aprendizaje de tares sencillas pero sí a tareas complejas, lo cual sugiere que el sueño paradójico es un factor especial no sólo para consolidar los aprendizajes sino también para un sueño cualitativo.

La denominación de actividad intelectual corresponde a un conjunto de actividades que movilizan los recursos de la persona para solucionar problemas, tareas o situaciones más o menos complejas. Se han estudiado las variaciones en la solución de problemas matemáticos, silogismos, rapidez y comprensión lectora, cálculo mental, concordancias verbales, entre otras tareas o situaciones a resolver. A la diversidad de tareas hay que añadir no sólo la incidencia de factores individuales y socioculturales, y el conocimiento que se tenga de los resultados que pueden modificar la estrategia a seguir para resolverlos, sino también la estrategia experimental y el criterio de valoración de los resultados (porcentaje, puntuación media, diferencia estadística...). Sin embargo, a pesar de estas dificultades existe un consenso entre los investigadores sobre la ciclicidad tanto circadiana como ultracircadiana de la actividad intelectual.

Ya se ha mencionado el trabajo de Klein et Armitage (1979) sobre la actividad rítmica y en fase opuesta de los dos hemisferios cerebrales de conformidad con el tipo de tarea a realizar. 
Otro aspecto de interés, lo presenta el considerar el cambio de estrategia durante la ejecución de la tarea: pasa de un control mínimo de automatización de la tarea a un control que exige una atención mantenida. Testu (1987) mediante la administración cuatro veces al día de una tarea matemática a sus alumnos, contrasta los resultados obtenidos en un grupo cuya tarea de resolución del problema matemático se automatiza (utiliza la misma estrategia), con los obtenidos por otro grupo que debía estar atento al problema puesto que exigía diferentes estrategias de solución. Los resultados del grupo de automatización de la solución muestran u incremento en las primeras horas para quedar estabilizado después y desaparece la ritmicidad. El grupo que ha de utilizar diferentes estrategias presenta un ritmo circadiano en sus resultados: incremento estabilidad y disminución. El tipo de actividad en la resolución del problema puede explicar la ritmicidad de la actividad intelectual solicitada en dicha tarea, pero también es posible que la capacidad de discriminación de la solución a aplicar sea debido a la incidencia del criterio de decisión o tal vez a la seguridad o convencimiento que se tiene al dar la solución.

\section{Conclusiones}

El conjunto sintético de datos expuestos explicita la compleja integración de los diversos factores que intervienen en nuestra actividad. El primero de ellos es el biológico, como lo ha evidenciado la Cronobiología y recientemente la neurobiología. El segundo, la variable edad o desarrollo y maduración de la persona, al hallarse en constante cambio. El tercero, las características del propio sujeto: personalidad, motivación, actitud e interés por la tarea. Un cuarto factor lo constituye el contexto sociocultural. Por último, y no menos importante, se refiere al investigador: la planificación, desarrollo de la investigación e interpretación de resultados.

\section{Referencias bibliográficas}

Acuña Castroviejo, Darío (2018). Sueño y Vigilia. Barcelona: EMSE EDAPP, S.L.

Agostino, Patricia V.; Plano Santiago A. y Golombek Diego A. (2007): "Sildenafil Accelerates Reentrainment of Circadian Rhythms". PNAS. Proceedings National Academy of Sciences of the United States of America, 104 (23), 9834-9839. DOI: https://doi.org/10.1073/pnas.0703388104.

Akerstedt, Torbjörn y Fröberg, Jan E. (1976): "Interindividual Differences in Circadian Patterns of Cathecolamine Excretion, Body Temperatura, Performance and Subjective Arousal". Biological Psychology, 4, 277-292.

Beugnet-Lambert, Claire (1985). Vigilance et cognition: aproche chronopsychologique de l'attention. Thèse doctorat de Psychologie. Lille: Université de Lill3.

Beugnet-Lambert, Claire; Lancry, Alain y Leconte, Pierre (1988): “Chronopsychologie. Rythmes et activités humaines". Lille: Presses Universitaires de Lille3.

Blake, M.J. F. y Corcoran, D. W.J. (1971): “Introversion-Extroversion and Circadian Rhythms” en W.P. Colcuhoun (ed.): Biological Rhythms and Performance. London: Academic Press.

Bloch, Vincent (1973): “Les niveaux de vigilance et l'attention” en Paul Fraisse y Jean Piaget (ed.): Traité de Psychologie Expérimentale. París: Presses Universitaires de France (PUF). 
Bloch, Vincent; Denti, A.y Schmaltz, G. (1966): "Effets de la stimulation réticulaire sur la phase de consolidation d'un apprentissage à essai unique par estimulation réticulaire". Journal of Physiology, 58, 469-470.

Bloch, Vincent; Deweer, Bernard y Hennevin, Elisabeth (1970): "Suppression de l'amnésie rétrograde et consolidation d'un apprentissage à essai unique par stimulation réticulaire". Physiology and Bebaviour, $5(11), 1235-1241$.

Canals, R.; Añaños, E. y Martín, M. (1989): "Ritmes circadians de l'atenció en una mostra d'alumnes de cicle mitjà”. Butlletí Universitari de Psicologia, IV (5), 13-15.

Crépon, Pierre (1983). Les rythmes de vie de l'enfant. París: Retz.

Folkard, Simon (1980): “A Note on 'Time of Day Effects in School Children's Immediate and Delayed Recall of Meaningful Material' - the Influence of the Importance of the Information Tested”. British Journal of Psychology, 71, 95-97.

Folkard, Simon; Bradury, Rosamund y Rosenthall, Joanna (1977): "Time of Day Effects in School Children's Inmediate and Delayed Recall of Meaning Full Material”. British Journal of Psychology, 68 (1), 45 50. DOI: https://doi.org/10.1111/j.2044-8295.1977.tb01557.x.

Gates, A. I. (1916): "Variations in Efficiency During the Day, Together with Practice Effects, Sex Differences and Correlations". University of California Publications in Psychology, 2, 1-156.

Hederich, Christian; Camargo, Ángela y Reyes, M.E. (2004). Ritmos cognitivos en la escuela. Bogotá: Universidad Pedagógica Nacional, División de Gestión de Proyectos, Centro de Investigaciones.

Hennevin, Elizabeth y Leconte, Pierre (1977): “Étude des relations entre le somneil paradoxal et les processus d'adquisition". Physiology and Behaviour, 18, 307-319.

Horne, James A. y Ostberg, Olov (1977): “Individual Differences in Human Circadian Rhythms”. Biological Psychology, 5 (3), 179-190. DOI: https://doi.org/10.1016/0301-0511(77)90001-1.

Horne, James A.; Brass, C. G. y Pettitt, A.N. (1980): “Circadian Performance Differences between Morning and Evening types”. Ergonomics, 23 (1), 29-36.

Hughes, D. G. y Folkard, Simon (1976): "Adaptation to an 8-h. Shift in Living Routine by Members of a Socially Isolated Community”. Nature, 264, 432-434.

Klein, R y Armitage, R. (1979): "Rhytms in Human Performance: 1:1/2 hour Oscillations in Cognitive Style”. Science, 204, 1326-1328.

Leconte, Pierre (1989). La Chronopsychologie. París: Presses Universitaires de France (PUF).

Leconte, Claire (2011). Des rythmes de vie aux rythmes scolaires. Villeneuve d'Ascq: Septentrion.

Meccaci, Luciano (1985). Radiografía del cerebro. Barcelona: Ariel.

Mills, J. N. (1964): "Circadian Rhythms During and after Three Months in Solitude Underground”, The Journal of Physiology, 174 (2), 217-231. DOI: https://doi.org/10.1113/jphysiol.1964.sp007483. 
Pöppel, E.; Aschoff, J. C. y Giedke, H. (1970): “Tagesperiodische Veränderungen der Reaktionszeit bei Wahlreaktionen”. Zeitschrift für Experimentelle und Angewande Psychologie, XVII, 537-552.

Roffwarg, Howard P.; Muzio, Joseph N. y Dement, William C. (1966): "Ontogenetic Development of the Human Sleep Dream Cycle”. Science, 152, 604-619.

Sáiz Roca, Dolores (1988). Una aproximación a los ritmos de la memoria. Tesis doctoral. Facultad de Filosofía y Letras. Universitat Autònoma de Barcelona. Bellaterra.

Sáiz Roca, Dolores y Estaún Ferrer, Santiago (1990): "A preliminary Studi of the Rhythms in Different Tasksof Memory" en E. Morgan (ed.): Chronobiology and Chronomedicine. Basic Researchand Applications. Frankfurt: Peter Lang.

Siffre, Michel (1963). Hors du temps. París: Julliard.

Testu, François (1987). Chronopsychologie et rythmes scolaires. Poitiers: Etude expérimentale.

Testu, François (1989). Chronopsychologie et rythmes scolaires. París: Masson.

Testu, François (1993). Etude des rythmes scolaires en Europe. París: Ministère de l'Education Nationale.

Thatcher, Robert W.; Walker, R. A. y Giudice, S. (1987): "Human Cerebral Hemispheres Develop at Different rates and Ages". Science, 236, 1110-1113.

Vermeil, Guy (1976). La fatigue à l'école. París: ESP.

\section{Nota biográfica}

Santiago Estaún Ferrer es doctor en Psicología por la Université René Descartes (Paris V) y catedrático emérito de Psicología de la Percepción y Psicología y Tiempo en la Universitat Autònoma de Barcelona. Ha publicado más de un centenar de artículos sobre Psicología y Tiempo y Percepción. Ha participado en numerosos congresos nacionales y internacionales de Psicología y Cronobiologia. 